\title{
Flying object tracking and classification of military versus nonmilitary aircraft
}

\author{
Ehsan Akbari Sekehravani ${ }^{1}$, Eduard Babulak ${ }^{2}$, Mehdi Masoodi ${ }^{3}$ \\ ${ }_{1,3}$ Young Researchers and Elite Club, Iran \\ ${ }^{2}$ Department of Computer Science, Liberty University, USA
}

\section{Article Info \\ Article history: \\ Received Oct 16, 2019 \\ Revised Feb 3, 2020 \\ Accepted Mar 15, 2020}

\section{Keywords:}

Aircraft

Flying object

Flying object tracking

Object detection

Particle filter

\begin{abstract}
Tracking of moving objects in a sequence of images is one of the important and functional branches of machine vision technology. Detection and tracking of a flying object with unknown features are important issues in detecting and tracking objects. This paper consists of two basic parts. The first part involves tracking multiple flying objects. At first, flying objects are detected and tracked, using the particle filter algorithm. The second part is to classify tracked objects (military or nonmilitary), based on four criteria; Size (center of mass) of objects, object speed vector, the direction of motion of objects, and thermal imagery identifies the type of tracked flying objects. To demonstrate the efficiency and the strength of the algorithm and the above system, several scenarios in different videos have been investigated that include challenges such as the number of objects (aircraft), different paths, the diverse directions of motion, different speeds and various objects. One of the most important challenges is the speed of processing and the angle of imaging.
\end{abstract}

This is an open access article under the CC BY-SA license.

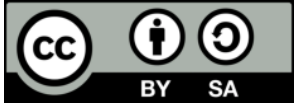

\section{Corresponding Author:}

Ehsan Akbari Sekehravani,

Young Researchers and Elite Club, Iran.

Email: akbarisekkehravani@gmail.com

\section{INTRODUCTION}

In addition to fixed objects, the real world often contains moving one. Hence, natural visual systems sensed and processed a continuous stream of observed scene data, and are less likely to encounter the perception of quite robust images. There is a similar situation in the machine vision. The visual system is often faced with a changing world that needs to be perceived and absorbed by successive images. Among the issues of interest in this regard is the question of tracking the motion of objects in a sequence of images. In this case, the visual system tries to detect the motion of objects on the scene by detecting sequential scenes from the scene during the period and identifying their movement paths. Object tracking is a significant field in computer vision [1]. The development of powerful computers, the availability of quality and cheap cameras, and the growing need for automotive video analytics has made a lot of attention to tracking algorithms for objects. Nowadays, visual aircraft detection is one of the interesting subjects in image processing projects because of the wide usage of low distance and low altitude aircraft.

Several object tracking techniques have been suggested [2-6] in the video surveillance context. The oldest and most well-known is the Kalman filter. More latterly, several increasingly advanced methods were used-including particle filters [7-11] Zhou et al. [12] uses the integration of color feature and contour knowledge in the particle filter based multi-hypothesis tracking algorithm. To enhance tracking performance unscented particle filter is a perfect choice to create relative distribution [13]. Iterative algorithms such as particle swarm optimization (PSO) [14] are suitable to be implemented in tracking problems. Indoor tracking 
was implemented based on radio signal strength indication [15]. The paper aims are to provide an algorithm for tracking flying objects in video images and then classify the type of tracked objects. In other words, identifying that classified objects are military or nonmilitary aircraft?. The paper is organized as follows. Detection and tracking of flying objects are presented in section 2. Section 3 is about the proposed classification type of flying objects (military or nonmilitary). The system simulation tool is introduced in section 4 . We present experimental results in section 5 and the conclusion in section 6 .

\section{FIRST PART: DETECTION AND TRACKING OF FLYING OBJECT}

Video analysis is the main task of a computer vision system that is used in many fields, including video surveillance, traffic monitoring systems, communication between humans and computers, maritime navigation, and so on. The analysis of video images consists of three basic steps:

- $\quad$ Detect the moving object.

- Follow the path of this object in sequential frames.

- An object motion path analysis to identify its behavior, object type detection, and the issuance of required control commands.

In the process of detecting objects by computer, interpretive elements are transformed into mathematical relations and implemented in the form of specific algorithms on the images. The important point in this discussion is the selection of appropriate features or parameters for extracting target information since the rest of the algorithm will create and overshadow this path. The question that is posed here is whether they are suitable features or how to choose a suitable set of features among the available features. It is very important to note that the function of detecting the system depends on the way objects are displayed. If the display of objects in a system is more accurate and based on more detailed information and features, then, that system will have more diagnostic power. Therefore, there is a determinant factor in choosing the power of display or resolution of existing data. On this basis, what is the process of discovery of the object and, consequently, the camouflage evaluation, operates so far as to be very dependent on this subject [16].

One of the first steps in processing an image is the image segmentation, which is one of the most difficult and most important stages in image processing. The segmentation of image processing has been considered widely in most branches of science and industry, and in many of these branches, the identification of the main components of the image is of great importance. For example, the automatic detection and tracking of moving objects in military applications and the separation of various products in industrial applications can be referred to as segmentation applications of the image.

Detection is a fundamental stage for analyzing video images that are involved with the segmentation and separation of moving objects on the scene. This section not only focuses on the processing of the next steps but also significantly reduces the time required for computing. Subtraction from sequential frames, background subtraction, and optical flow are the most widely used methods for detecting. Due to environmental conditions such as camera movement, noise, lighting changes, shadows and repetitive movements of some parts of the background, such as branches of trees, the partitioning of moving objects is a relatively difficult problem, and its proper implementation is of considerable importance. In [17], they proposed a particular tracking algorithm for airplane based on R-FCN and kalman filter (KF). The great detection network, R-FCN, was used to provide the position message of a plane for the tracking model.

\subsection{Proposed tracking algorithm}

After discovering the purpose of the field and providing the appropriate presentation, it turns to track them. The purpose of tracking is to determine the position of objects in each image of the input sequence. To track objects, we need an algorithm to estimate the state of a dynamic system from incomplete and misleading measurements.

\subsection{Particle filter $(\mathbf{P F})$}

Nowadays, with the growth of methods including statistical methods, the problem of estimating a parameter or the state of a random nonlinear system along with observation and noise measurements is performed by the Monte Carlo estimation technique for solving complex statistical problems, which is expressed in the form of a particle filter algorithm [18]. As KF is only limited to the Gaussian or normally distributed state variables and estimate them very poorly. This problem is overcome by the particle filter. Particle filter as used in $[19,20]$, can estimate the internal states from partial observations. This method is suitable for non-Gaussian and multi-model possibility density function. The particle filter is a new method for obtaining the probability distribution function based on the Bayesian theory. The particle filter algorithm is based on Monte Carlo (MC) chain methods in which a probability density particle representation is used to estimate the parameters of an arbitrary distribution. The particle filter is the perfect solution for optimal 
purpose estimation in a situation where the nonlinear system model is non-Gaussian with the distribution of noise. The system's transition or movement model is expressed in terms of probability language, $\mathrm{p}\left(\mathrm{x}_{\mathrm{t}} / \mathrm{x}_{\mathrm{t}-1}\right)$, and the model of the observation or measurement of the system is expressed by $\mathrm{p}\left(\mathrm{x}_{\mathrm{t}} / \mathrm{z}_{\mathrm{t}-1}\right), \mathrm{x}_{\mathrm{t}}$ representing the system state vector in the current $Z_{t}$ and representative view in the system. Considering the Bayesian recurrence equations that are made up of the following two steps [18]:

Prediction stage:

$$
\mathrm{p}\left(\mathrm{x}_{\mathrm{t}} / \mathrm{z}_{\mathrm{t}-1}\right)=\int_{\mathrm{R}^{\mathrm{n}}} \mathrm{p}\left(\mathrm{x}_{\mathrm{t}} / \mathrm{x}_{\mathrm{t}-1}\right) \mathrm{p}\left(\mathrm{x}_{\mathrm{t}-1} / \mathrm{z}_{\mathrm{t}-1}\right) \mathrm{dx} \mathrm{x}_{\mathrm{t}-1}
$$

Update stage:

$$
\begin{aligned}
& p\left(x_{t} / z_{t}\right)=\frac{p\left(z_{t} / x_{t}\right) p\left(x_{t} / z_{t-1}\right)}{p\left(z_{t} / z_{t-1}\right)} \\
& p\left(x z_{t} / z_{t-1}\right)=\int_{R^{n}} p\left(\frac{z_{t}}{x_{t}}\right) p\left(\frac{x_{t}}{z_{t-1}}\right) d x
\end{aligned}
$$

The obtained models are usually non-linear and probabilistically possible to have non-Gaussian distributions and even multiples. One of the most well-known methods for solving the filtration problem is the EKF method. Since this method uses the Taylor series estimator to use a nonlinear function, it has some error. One of the methods that can be used in such cases is estimated by particle filters. Integrating action takes place in the process of state-space variables, regardless of whether such an integral can be in any closed state, if the size of the space is high, it will have a high computational cost. The Monte Carlo simulation method, instead of calculating the integral overall points, performs its sampling at the points that have the largest share in the computation of the integral. The basis for this is to calculate the integral:

$$
I=\int g(x) d x
$$

Converts it as a product of the term $g(x)=f(x) \cdot p(x)$ where $p(x)$ represents a probability function that satisfies the condition $\left\{\int \mathrm{p}(\mathrm{x}) \mathrm{dx}=1\right.$ and $\left.\mathrm{p}(\mathrm{x}) \geq 0\right\}$, accordingly, with these conditions $I$ is an Expected value of the optional function $\mathrm{f}(\mathrm{x})$. Now, if the model of the system state up to moment $\mathrm{k}$, expressed by the function $\mathrm{p}\left(\mathrm{X}_{\mathrm{k}} / \mathrm{Z}_{\mathrm{k}}\right)$, is sampled independently of N's number of particles $(\mathrm{N}>>1)$ and distribution such as $\left(\mathrm{x}_{0: \mathrm{k}}^{\mathrm{i}}\right)$ [18]:

$$
;(\mathrm{i}=1.2 \cdots \cdot \mathrm{N}) \mathrm{X}_{\mathrm{k}}^{\mathrm{i}} \sim \mathrm{p}\left(\mathrm{X}_{\mathrm{k}} \mid \mathrm{Z}_{\mathrm{k}}\right)
$$

The estimated distribution of this distribution is defined as follows:

$$
\mathrm{P}_{\mathrm{N}}\left(\mathrm{X}_{\mathrm{k}} / \mathrm{Z}_{\mathrm{k}}\right) \cong \frac{1}{\mathrm{~N}}
$$

Where $\delta\left(\mathrm{X}_{\mathrm{k}}-\mathrm{X}_{\mathrm{k}}^{\mathrm{i}}\right)$ represents the Dirac Delta function at a point $\left(\mathrm{X}_{\mathrm{k}}-\mathrm{X}_{\mathrm{k}}^{\mathrm{i}}\right)$. Such an estimate is also used to distribute filtering. If the Expected value of the desired function $f(0)$ is represented by I respect to $p\left(X_{k} / Z_{k}\right)$ :

$$
\mathrm{I}=\int \mathrm{f}\left(\mathrm{X}_{\mathrm{k}}\right) \cdot \mathrm{p}\left(\mathrm{X}_{\mathrm{k}} / \mathrm{Z}_{\mathrm{k}}\right) \mathrm{d} \mathrm{X}_{\mathrm{k}} \approx \int \mathrm{f}\left(\mathrm{X}_{\mathrm{k}}\right) \cdot \mathrm{P}_{\mathrm{N}}\left(\mathrm{X}_{\mathrm{k}} / \mathrm{Z}_{\mathrm{k}}\right) \mathrm{d} \mathrm{X}_{\mathrm{k}}
$$

Then, the Monte Carlo estimation of the integral as the sum of the values of the function $\mathrm{f}$ for the obtained samples is as follows:

$$
\mathrm{I}_{\mathrm{N}}^{\wedge}=\frac{1}{\mathrm{~N}} \sum_{\mathrm{i}=1}^{\mathrm{N}} \mathrm{f}\left(\mathrm{X}_{\mathrm{k}}^{\mathrm{i}}\right) v
$$

If samples $\mathrm{X}_{\mathrm{k}}^{\mathrm{i}}$ are independent, then $\mathrm{I}_{\mathrm{N}}$ is an unbiased estimate and can be approximated by the law of large numbers with $\mathrm{I}$, and if $\mathrm{N}$ is a very large number, the estimate will converge to the real value. 
It is also shown that the convergence rate of estimation is independent of the variance and the dimensions of the characteristic space and depends only on the number of particles of N. Such a characteristic is the most important advantage of Monte Carlo integral to numerical integration methods [18]. In this paper, the particle filter algorithm predicts the position of the $N$ particle in the range around the center of the mass of the previous frame randomly to begin to trace the particle. Each particle can be nominated for a new target position. By applying a kernel similar to the target model, the weighted histogram of the candidate particle model is obtained for each particle. The Bhattacharyya coefficient calculates the similarity between the object model and the object candidate particle model. The likelihood function detects particle visibility to enter the open sampling stage. The initial position of the moving object is estimated after the number of adaptive stages of the re-entry.

\section{SECOND PART: CLASSIFICATION TYPE OF FLYING OBJECTS (MILITARY OR NONMILITARY)}

It is worth remembering that in designing a system for classifying and categorizing an object, it is necessary to have a set of input data belonging to each desired category. From another point of view, it is possible to designate a system in such a way as to deal with an object belonging to one of the categories defined in terms of one or more specified categories and collecting the collection of their respective images, select it carefully to the category, and otherwise declare it to be an unknown or invalid object. The advantage of this method is that the system is reliable in categorizing valid objects that belong to one of the categories defined, logical coherence is observed among the members of each category, and more importantly, the description of the objectives is classified as desirable. In other words, according to the existing facilities and limitations, and the definition of several specific categories, specific and proportionate to the application, it is applied to system design. Since the further separation of objects into a classification, in addition to a more complete description of the target's purpose, is the ability and efficiency of the designed system. The selected categories of this paper are selected according to Figure 1.

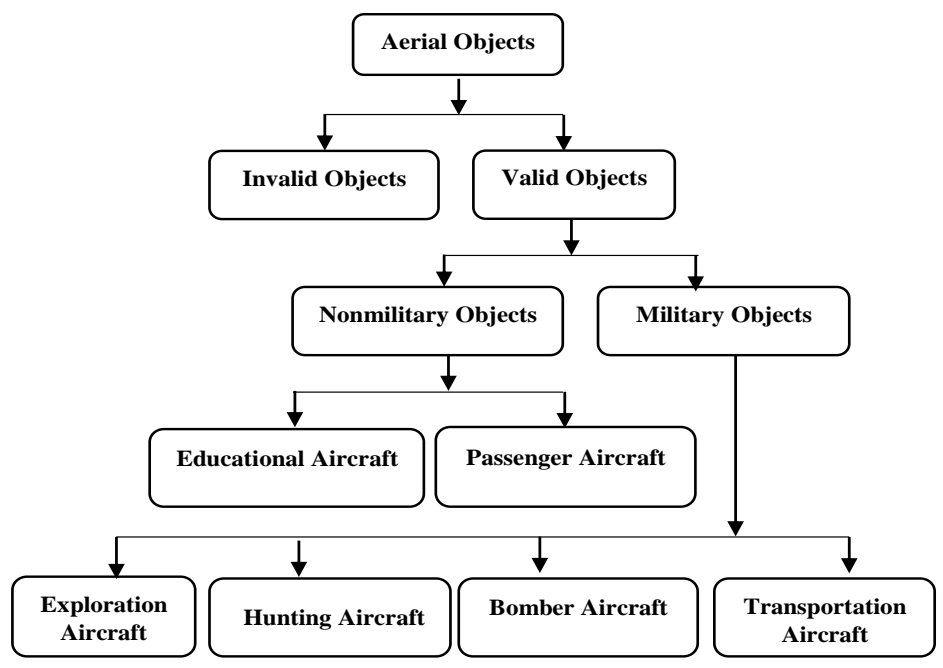

Figure 1. Categories of flying objects for designing this paper

Therefore, in this paper, the two categories of valid objects and invalid objects are the desired outcomes of the system. In other words, the system is expected to be assigned to any flying object in contact with any of the defined categories, or to declare it categorized as inappropriate. Considering the above categories means that a collection of appropriate and comprehensive images as educational data of each category is available.

\subsection{Aircraft type detection system (military or nonmilitary)}

First, tracking flying objects that were done by the particle filter algorithm. Then, the tracked objects by the aircraft type detection system (military or nonmilitary aircraft), are investigated through four criteria including: size (center of mass) of objects, object speed vector, the diverse direction of motion of objects and thermal imagery. Ultimately, the type of flying objects (military or nonmilitary aircraft) is determined. Four criteria for the aircraft type detection system are shown in Figure 2. 


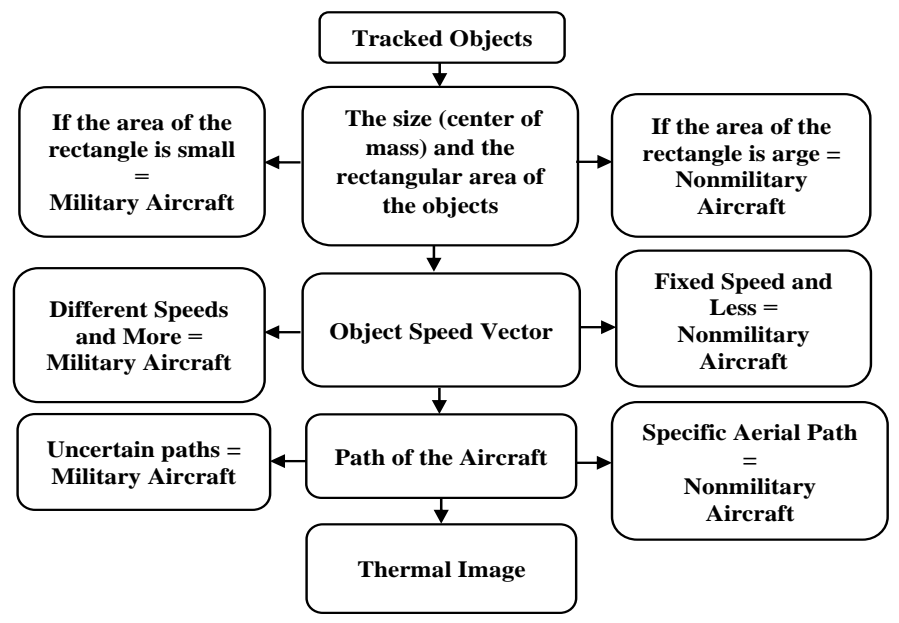

Figure 2. Flowchart aircraft type detection system

\subsection{Size (center of mass) of object}

First, it is needed to have binary images and objects are identified from the background. To obtain the center of the mass of the moving object, we need to sum up the quantities of $\mathrm{x}$ and $y$ that are white in relation to the object and divide them into numbers [21]. In this way, the coordinates and position of the moving object are obtained. To track and identify changes, the moving object is detected and tracked by a rectangle with specified size or eight lines that radiate from the center of mass.

In this criterion, initially, we need to convert the images to binary and then get the size and position of the tracked objects in successive frames. The method works in such a way that in the full screen the areas of the object are 1 and the background is 0 . Then we compute the coordinates of the objects in the whole page (from top to bottom and from left to right) using (9) and (10) combine $\mathrm{x}$ together and $y$ together, in which the mean values of $\mathrm{x}$ and $y$ are obtained. Next, we get a total number of pixels. In the end to obtain the center of object mass using (11) we divide the average value of $\mathrm{x}$ and $y$ into the total pixels. In general, the total size, wings, and center of mass of nonmilitary aircraft are larger than military aircraft. It is likely that according to the coordinates, it was decided that the object is military or nonmilitary. The maximum $\mathrm{x}$ is the tip of the plane. Determining the center of mass is for this point to be a fixed point relative to the points on the edge. An example of obtaining a center of mass is shown in Figure 3.

$$
\begin{aligned}
& \bar{X}=\sum_{i=0}^{n} x_{i} \\
& \overline{\mathrm{Y}}=\sum_{\mathrm{i}=0}^{\mathrm{n}} \mathrm{y}_{\mathrm{i}}
\end{aligned}
$$

$$
\text { center of mass }=\frac{\overline{X . Y}}{\text { total number of pixels }}
$$

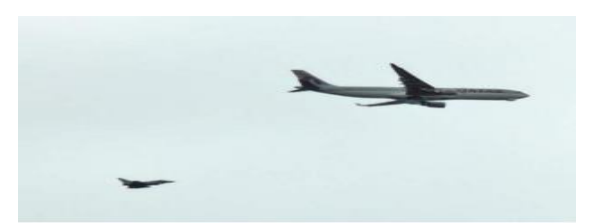

(a)

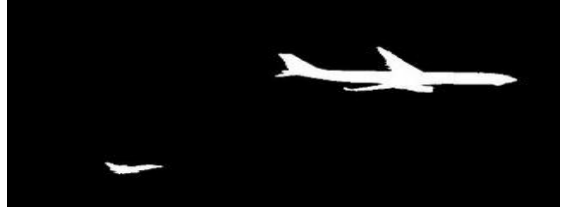

(b)

Figure 3. An example of obtaining a center of mass, (a) Original image, (b) Detected planes 


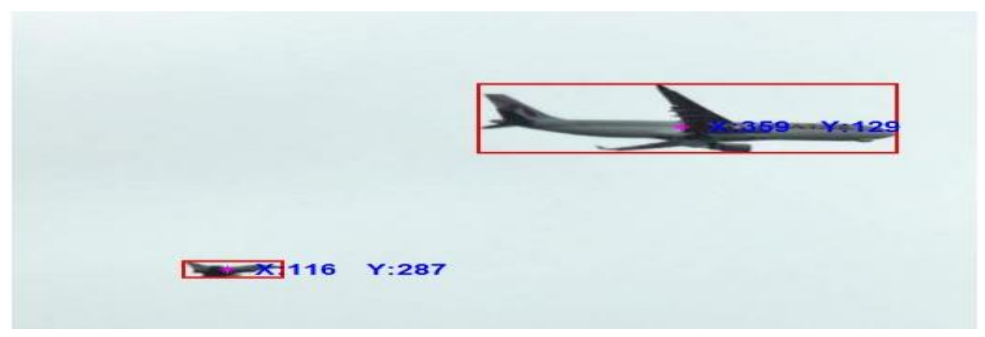

(c)

Figure 3. An example of obtaining a center of mass, (c) Coordinate and center of mass (Continue)

Figure 3 illustrates that there is a military aircraft and a nonmilitary aircraft in the frame, by obtaining the center of mass and the area of the aircraft rectangle, it can be concluded that the larger rectangular area of the object, is nonmilitary aircraft. Additionally, the rectangular area of the object, which is smaller, determines the military aircraft.

\subsection{Object speed vector}

At this stage, firstly, we need to measure the speed of the tracked objects in successive frames. Aircraft have different speeds. Nonmilitary aircraft such as regular passenger aircraft usually move at a steady pace, but military aircraft can travel at different speeds, the speed of these aircraft is much higher than the speed of nonmilitary aircraft. This feature can be used to detect a military aircraft from a nonmilitary when the object velocity decreases or increases over time, the object is the military aircraft, and if the object velocity is low and constant, the object is the nonmilitary aircraft. But the disadvantage of this method is that military aircraft can match their speed at the speed of nonmilitary aircraft, and it deceives the aircraft detection system. To calculate the object speed vector, first, we obtain the object position (x.y) in two different frames. Then, using (12), we obtain the Euclidean distance d between two different frames and, the object speed is obtained by inserting in (13). $\left(\mathrm{x}_{1} \cdot \mathrm{y}_{1}\right)$ and $\mathrm{t}_{1}$ are the position of the center of the object mass in the first frame and the time frame of the first frame, respectively. $\left(\mathrm{x}_{2} \cdot \mathrm{y}_{2}\right)$ and $\mathrm{t}_{2}$ are respectively, the position of the object mass center in the second frame and the time of the second frame.

$$
\begin{aligned}
& \text { Euclidean distance }=\mathrm{d}=\sqrt{\left(\mathrm{X}_{2}-\mathrm{X}_{1}\right)^{2}}+\left(\mathrm{Y}_{2}-\mathrm{Y}_{1}\right)^{2} \\
& \text { Object speed }=\mathrm{V}=\frac{\mathrm{d}}{\mathrm{t}_{2}-\mathrm{t}_{1}} \frac{\mathrm{m}}{\mathrm{s}}
\end{aligned}
$$

\subsection{Aircraft move path}

The main difference between military and nonmilitary aircraft is that nonmilitary aircraft travel on a specific path, while military aircraft move in different directions. By using this criterion, we can acquire flight paths. By obtaining a center of mass of objects in different frames, different points are obtained in each frame and by connecting these points, the direction of motion of the objects (aircraft) in the video, will be distinguished which can show the direction of movement of aircraft on a chart. Then it can be decided that aircraft that travel on a specific route are nonmilitary or military. The disadvantage of this criterion is that military aircraft can move in a certain direction and it makes the aircraft detection system deceived. Flight paths are one of the most important characteristics that can be considered for a flying object.

\subsection{Thermal imagery}

The range of thermal images is provided by imaging systems that have detectors that are sensitive to thermal radiation in the infrared electromagnetic spectrum. In general, imaging systems are a subset of electro-optic systems. Since an infrared heating system is responsible for preparing the input images of the system, familiarity with its fundamental concepts and structure, in general, can be effective in choosing the appropriate methods. The simulation of infrared thermal imagery is not possible as a solution to the problem of the rarity of heat-fitting images appropriate to the application, without being fully familiar with the structure of infrared imaging systems [22].

Thermal cameras and imaging have periodically been in interest mainly for smart environments, military applications. The increasing amount of image quality and resolution since the last few years have, however, unlock new challenges and application areas in thermal imagery. Thermal cameras are useful to 
measure a temperature difference. These cameras are helpful compared to cameras operating in the visual spectrum due to their ability to acquire images in the complete night, robust to lighting variations and less interruption on privacy, as tempering is tedious [23]. The detection and tracking of an airborne infrared target in a complex struggle situation remain a challenging research area in infrared-imaging guidance [24, 25]. This criterion is not effective because the camera used in this article is not a thermal camera.

\section{SYSTEM SIMULATION TOOL}

To implement the detection sector, tracking and object estimation are required for a suitable digital processor. Furthermore, virtual simulation of the scene and imaging system will be possible using programmable digital processors. In this paper, a fixed camera is used for imaging, and the videos we want to process on them are virtualized with fixed background and with a computer. Personal computers are one of the best environments for applications like this. The advantage of using a computer in the simulation of the system is that the implementation of the detection and tracking section along with the estimated part using the main processor of the computer and its memory is well suited. Moreover, rapid access to lateral information and multimedia of the computer will facilitate the processes of comparison, evaluation and information transfer with greater speed and accuracy. The characteristics of the computer that is selected as the virtual simulation laboratory of the system are according to Table 1 . The processor's programming and execution of the computational methods are based on the environment and facilities available in MATLAB software. The use of MATLAB software for its appropriate toolbox for processing digital data and image processing, as well as the ease of file management in it, has been considered as a virtual environment for simulating the system.

Table 1. Computer system specifications used as a virtual laboratory

\begin{tabular}{ll}
\hline \multicolumn{1}{c}{ Specifications } & \multicolumn{1}{c}{ Components } \\
\hline Microsoft Windows 10 Enterprise & Operating System \\
4.00GB (3.89GB usable) & RAM \\
Intel(R) Core(TM) i5-4210U CPU@ 1.70GHz 2.40GHz & CPU \\
ASUS X552L Graphic Card Intel(R) HD NVIDIA GeForce820M & Frame Grabber \\
MATLAB R2013a & Software \\
\hline
\end{tabular}

\section{EXPERIMENTAL RESULTS}

To demonstrate the efficiency and resilience of the algorithm and the above system, several scenarios in various videos including challenges such as the number of objects (aircraft), the different paths, the direction of motion, the different speeds and various objects, have implemented. It is necessary to explain that in the forms of aircraft images, they are used for detecting and tracking the object. The yellow rectangle is pulled around the tracked objects, the red mark $\left(^{*}\right)$ indicates the real center of the object, which is extracted from the center of mass by the modeling method and the criterion of the center of mass, and the green star sign $\left(^{*}\right)$ represents the center of mass of an estimated object by particle filter as shown in Figure 4. The letters $(\mathrm{A} 1, \mathrm{~A} 2, .$.$) in the graphs indicate the direction of the movement of planes numbers (1,2, \ldots)$.

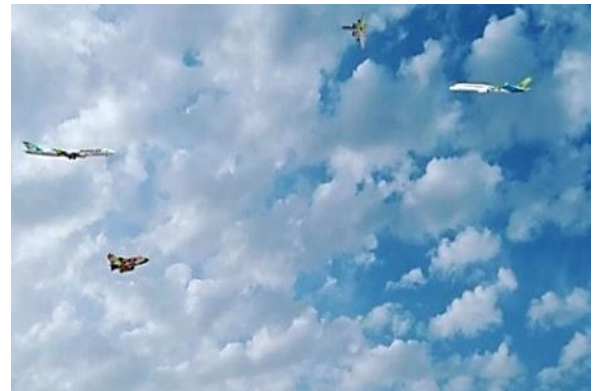

(a)

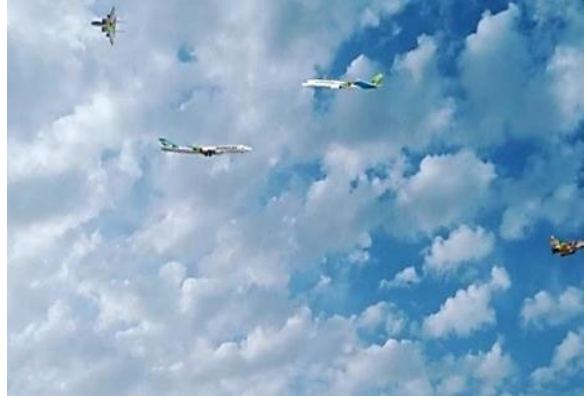

(b)

Figure 4. Calculates the center of mass in different frames, (a) Frame 58, (b) Frame 110 
Scenario: in the video, there are two passenger aircraft of different sizes, different directions, and two different military aircraft moving in different directions. Furthermore, the string of images has 219 image frames with dimensions of $480 \times 850$. Consequently, the objects in this sequence of images are constant in terms of reshaping and changing size. First, aircraft that are moving are detected and they are separated from the background, the objects are white and the background is black as shown in Figure 5. Figure 6 reveals that the particle filter algorithm could reliably track targets up to the end of the image string. The number of tracked objects is displayed as numbers 1, 2, and 3 above the targets. By calculating the center of mass and the area of the planet's rectangles: The area of aircraft numbers 2 and 3 is more than the aircraft 1 and 4. According to the results of the aircraft speeds in Table 2, aircrafts numbers 1 and 4 have a higher speed than aircrafts numbers 2 and 3. Figure 7 shows that the direction of the movement of aircrafts numbers 1, 2 and 3 are in a straight line, and the direction of aircraft number 4 is not a straight line. Hence, according to the results, we conclude that the aircraft number 1 and 4 are military aircraft and aircraft number 2 and 3 nonmilitary aircraft.

Table 2. Aircraft speed results

Speed Achieve $\frac{m}{s} \quad$ Aircraft Specifications

Number 1

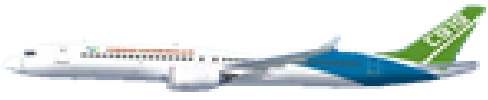

Number 2

119

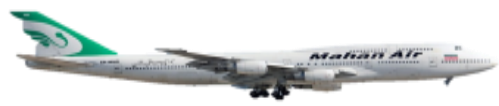

Number 3

368

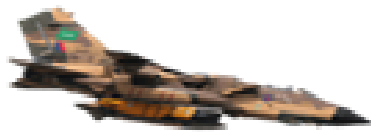

Number 4

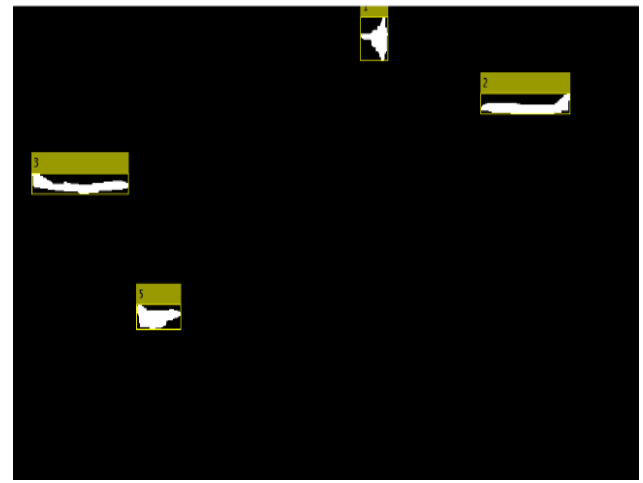

(a)

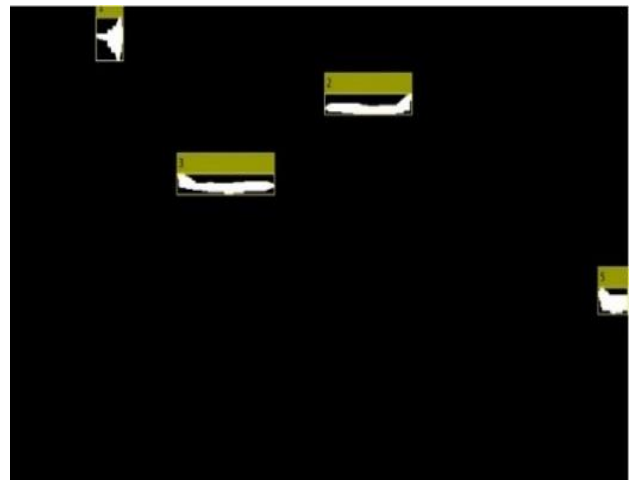

(b)

Figure 5. Objects detection in different frames, (a) Frame 58, (b) Frame 110 


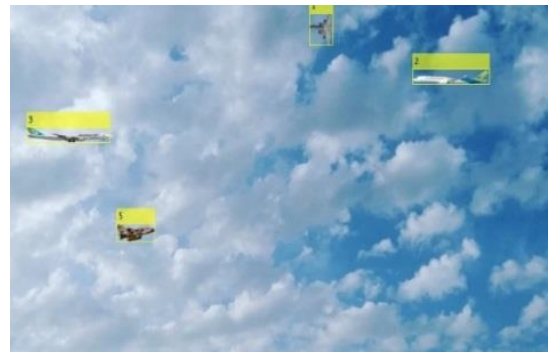

(a)

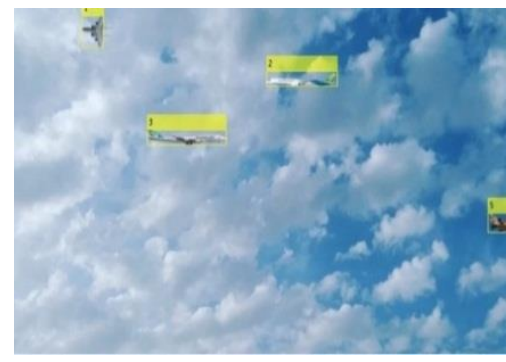

(b)

Figure 6. Objects tracking by particle filter in different frames, (a) Frame 58, (b) Frame 110

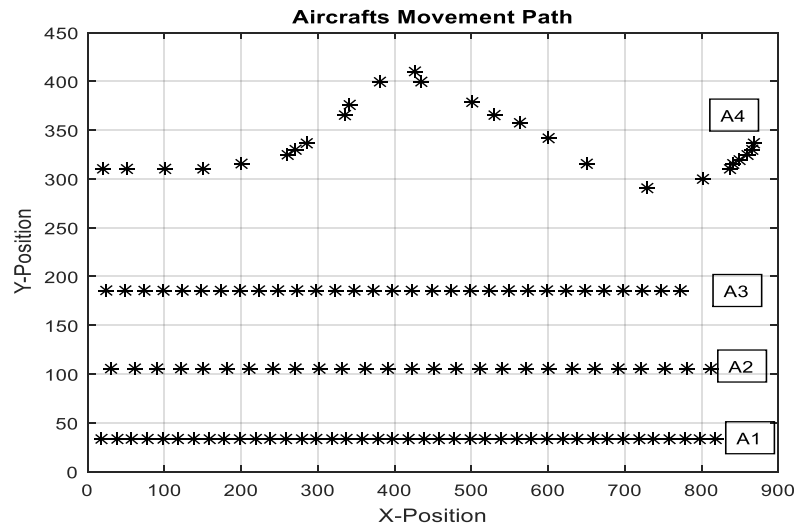

Figure 7. Aircraft movement paths

\section{CONCLUSION}

In conclusion, this paper contains two main parts, the first part is the detection and tracking of flying objects. At first, the aircraft that are moving are detected and separated from the background, the object is white and the background is black. Flying objects are tracked using the particle filter algorithm. The particle filter algorithm predicts the position of $\mathrm{N}$ particle in the range around the center of mass of the previous frame randomly to start tracking. Each particle can be nominated for a new target position. The second part is to classify flying objects (military or nonmilitary), based on four criteria; Size (center of mass) of objects, object speed vector, the direction of motion of objects, and thermal imagery identifies the type of tracked flying objects. Detection and tracking based on the proposed method and aircraft type detection system have been implemented on a large number of images. To demonstrate the efficiency and resilience of the algorithm and the above system, several scenarios in various videos have been tested that include challenges such as the number of objects (aircraft), the different paths, the divers' directions of motion, different speeds and various objects. In this paper, only the results of one scenario are mentioned. According to the results of the implementation of the proposed system, detection, tracking and aircraft type detection system for a variety of different images has been adequately answered.

\section{REFERENCES}

[1] B. Y. J. L. Z. e. a. Jiang, "Wearable assistant vision system for visually impaired users based on binocular sensors," in IEEE Internet Things J, pp. 1, 2018.

[2] C. K. a. C. H. a. R. Nevatia, "Multi-target tracking by online learned discriminative appearance models," in In the IEEE CVPR, San Francisco, CA, USA, pp. 685-692, June 2010.

[3] K. S. a. L. V. G. B. Leibe, "Coupled detection and trajectory estimation for multi-object tracking," In ICCV, pp. 1-8, 2007.

[4] A. P. L. a. S. Gong, "Mean-shift tracking with random sampling," in In British Machine Vision Conference $(B M V C)$, Edinburgh (UK), September, 2006.

[5] L. S. a. I. V. a. G. Foresti, "Dynamic models for people detection and tracking," In the 8th IEEE AVSS, pp. 29-35, Sep 2008.

[6] A. Y. a. O. J. a. M. Shah, "Object tracking: A survey," vol. 38, no. 4, pp. 1-45, Dec 2006.

[7] D. S. a. A. S. N. Gordon, "Novel approach to non-linear/non-Gaussian Bayesian state estimation," IEEE Proceedings-F, vol. 140, no. 2, pp. 107-113, 1993. 
[8] M. I. a. A. Blake, "Condensation-conditional density propagation for visual tracking," IJCV, vol. 29, no. 1, pp. 5-28, 1998.

[9] G. Kitagawa, "Monte Carlo filter and smoother for nonGaussian nonlinear state space models," Journal of Computational and Graphical Statistics, vol. 5, no. 1, pp. 1-25, 1996.

[10] A. T. N. D. F. J. L. a. D. L. K. Okuma, “A boosted particle filter: Multitarget detection and tracking,” In ECCV, 2004.

[11] A. D. a. P. P. J. Vermaak, "Maintaining multimodality through mixture tracking," In ICCV, pp. 1110-1116, 2003.

[12] Bing Pu ,Fugen Zhou ,Xiangzhi Bai, "Particle Filter Based on Color Feature with Contour Information Adaptively Integrated for Object Tracking," in Fourth International Symposium on Computational Intelligence and Design, 2011.

[13] Y. Rui and Y. Chen, "Better Proposal Distributions: Object Tracking Using Unscented Particle Filter," in In the IEEE CVPR, Kauai, HI, USA, pp. II-II, 2001.

[14] Mehdi Masoodi, Mohsen Maesoumi, Ehsan Akbari Sekehravani, "Low Complexity Power Allocation Scheme In Cognitive Radio Sensor Networks To Raise Energy Efficiency," Far East Journal of Electronics and Communications, vol. 18, pp. 251-267, 2018.

[15] Mehdi Masoodi, Ehsan Akbari Sekehravani, Mohsen Maesoumi, "Rssi-Based Modified K-Nearest Neighbors Algorithm For Indoor Target Tracking," Far East Journal of Electronics and Communications, vol. 18, pp. 345-356, 2018.

[16] R. S. Robert A.Schowengerdt, "Models And Methods For Image Processing Academic Press," 2006

[17] W. Z. Y. H. C. J. B. J. Z. Z. H. S. Jiachen Yang, "Aircraft tracking based on fully conventional network and Kalman filter," The Institution of Engineering and Technology, vol. 13, no. 8, pp. 1259-1265, May 2019.

[18] F. N. D. a. G. N. Doucet. A, "Sequential Monte Carlo methods in practice," in Springer, New York, 2001.

[19] L. C. F. L. a. Y. J. L. ChongYi, "Target tracking based on extended Kalman particle filter," in 3rd IEEE International Conference on Computer and Communications (ICCC), Chengdu, pp. 1715-1719, 2017.

[20] L. Fu, "Particle Filter Pedestrian Tracking Algorithm Based on Selected Region RGB Histogram," in 2018 International Conference on Robots \& Intelligent System (ICRIS), Changsha, China, pp. 287-290, 2018.

[21] M. O. a. U. Deniz, "Center of mass model: A novel approach to background modeling for segmentation of moving objects," Image Vis.Comput, vol. 25, pp. 1365-1376, Aug. 2007.

[22] E. Nick, “A Practical Introduction Using Java," in Digital Image Processing, 2000.

[23] L. P. Harsh Motka, "A Vision Based Approach For Anomaly Detection In Smart Environments Using Thermal Images," International Journal of Innovative Technology and Exploring Engineering, vol. 8, no. 7, May, 2019.

[24] S. Wu, S. Niu, K. Zhang and J. Yan, "An Aircraft Tracking Method in Simulated Infrared Image Sequences," in In Pacific-Rim Symposium on Image and Video Technology; Springer, Cham, Switzerland, 2019.

[25] M. Wan, G. Gu, W. Qian, K. Ren, Q. Chen, H. Zhang and X. Maldague, "Total Variation Regularization TermBased Low-Rank and Sparse Matrix Representation Model for Infrared Moving Target Tracking," in Remote Sens, 2018

\section{BIOGRAPHIES OF AUTHORS}

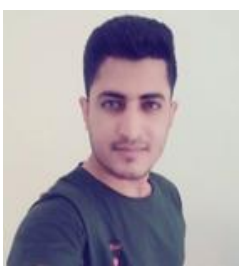

Ehsan Akbari Sekehravani was born in Iran in 1990. He received the M.S.c degree in Telecommunications Engineering from Islamic Azad University, Jahrom Branch, Iran, in 2016. $\mathrm{He}$ is currently a Ph.D. student in Electromagnetic engineering at the University of Campania Luigi Vanvitelli, Aversa, Italy. His main fields of interest are Image processing, Signal processing, Inverse Scattering problems, and Inverse source problems.

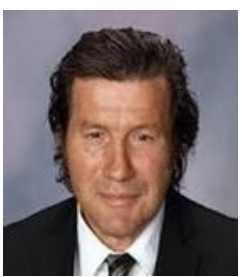

Professor Eduard Babulak received the D.Sc. degree from the University of Pardubice (Czech Republic), 2008 and also the Ph.D. degree from the Staffordshire University (United Kingdom), 2003. He is currently a Professor of Computer Science and Cyber Security, Liberty University, USA. He is an accomplished international scholar, researcher, consultant, educator, professional engineer and polyglot, with more than thirty years of experience.

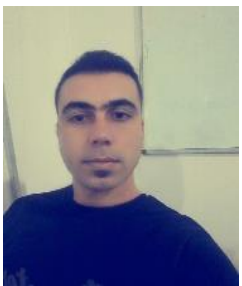

Mehdi Masoodi was born in Iran in 1987. He received the M.S.c degree in Telecommunications Engineering from Islamic Azad University, Kazerun Branch, Iran, in 2015. He is currently a Ph.D. student in Electromagnetic engineering at the University of Campania Luigi Vanvitelli, Aversa, Italy. His main fields of interest are Signal processing, Image processing, Inverse Scattering problems, and Inverse source problems. 